Recherches en didactique des langues et des cultures

Les cahiers de l'Acedle

17-2 | 2020

Recherches collaboratives en didactique des langues

\title{
Orientations méthodologiques et théoriques d'une recherche collaborative sur le travail documentaire de professeures d'anglais langue étrangère
}

\section{Margaret Bento}

\section{(2) OpenEdition}

Édition électronique

URL : https://journals.openedition.org/rdlc/7284

DOI : $10.4000 /$ rdlc.7284

ISSN : 1958-5772

Éditeur

ACEDLE

Référence électronique

Margaret Bento, «Orientations méthodologiques et théoriques d'une recherche collaborative sur le travail documentaire de professeures d'anglais langue étrangère », Recherches en didactique des

langues et des cultures [En ligne], 17-2 | 2020, mis en ligne le 27 avril 2020, consulté le 17 octobre 2022. URL : http://journals.openedition.org/rdlc/7284 ; DOI : https://doi.org/10.4000/rdlc.7284

Ce document a été généré automatiquement le 17 octobre 2022.

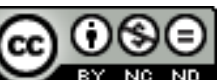

Creative Commons - Attribution - Pas d'Utilisation Commerciale - Pas de Modification 4.0 International - CC BY-NC-ND 4.0

https://creativecommons.org/licenses/by-nc-nd/4.0/ 


\title{
Orientations méthodologiques et théoriques d'une recherche collaborative sur le travail documentaire de professeures d'anglais langue étrangère
}

\author{
Margaret Bento
}

\section{Introduction}

1 Les articles rendant compte de recherches collaboratives ou participatives sont de plus en plus présents dans le paysage de la didactique des langues (entre autres NarcyCombes, 2005 ; Demaizière et Narcy-Combes, 2007 ; Macaire, 2007a ; Castellotti, 2013). Ces orientations impliquent une nouvelle manière de faire de la recherche « qui va dans le sens de valoriser un processus de production des connaissances réalisé de concert avec les acteurs concernés » (Anadon, 2007 : 3). Pour Desgagné (1997 : 371) :

\footnotetext{
«l'approche collaborative suppose une démarche de coconstruction entre les partenaires concernés; elle joue sur deux registres à la fois, soit celui de la production de connaissances et celui du développement professionnel des praticiens; elle contribue au rapprochement, voire à la médiation entre communauté de recherche et communauté de pratique. À l'horizon de ces trois énoncés se profile une vision socioconstructiviste du «savoir» à développer, et cela, à partir d'une démarche collective d'interprétation où théorie et pratique sont constamment mises en relation ».
}

2 Ainsi, on voit se mettre en place des collaborations complémentaires entre chercheurs et enseignants (Bednarz, 2013) et leurs conséquences sur l'environnement institutionnel dans lequel s'inscrivent ces recherches, souvent recherches-actions (voir par exemple la rétrospective de 5 programmes de recherches collaboratives en langues modernes de Beckers et Simons, 2010: 31-46 ou encore, Macaire, 2007a: 93-120), 
parfois recherches-interventions (Narcy-Combes, 2005 ; Demaizière et Narcy-Combes, 2007; Macaire, 2007b; Castellotti, 2013). Notons qu'un modèle de recherche collaborative implique de faire de la recherche avec les enseignants alors qu'une recherche-action ou intervention ne mettant pas en place de recherche collaborative porte sur les enseignants. Il y a donc une double fonction de recherche et de formation dans une approche collaborative.

3 Parallèlement, les recherches actuelles en didactique des langues s'intéressent peu aux pratiques effectives des enseignants quant à leur travail documentaire pour l'élaboration de séquences pédagogiques et notamment, leur point de vue est peu interrogé.

4 La présente recherche porte sur l'usage des ressources pédagogiques (documents audio, vidéo, écrits, iconographiques...) par des professeures d'anglais du secondaire en France. Cette étude entre dans le cadre plus large du projet ANR ReVEA (Ressources vivantes pour l'enseignement et l'apprentissage) portant sur l'usage des ressources par des enseignants du secondaire en France ${ }^{1}$.

5 On s'intéresse ici à la fois aux manières de didactiser, d'organiser le contenu des séquences pédagogiques, mais aussi à l'éthique professionnelle que ces choix engendrent ou qui est engendrée par ces choix.

6 L'implication des enseignantes comme actrices principales pour comprendre cet aspect de leur pratique m'a amenée à choisir une approche de recherche collaborative. Une activité réflexive lors de plusieurs rencontres avec ces professeures a servi de matériau d'analyse.

7 Dans cet article, je souhaite aborder les aspects méthodologiques et théoriques liés à une recherche qui tente de comprendre le travail de documentation d'enseignantes dans une approche collaborative et plus précisément comment s'effectue leur travail documentaire pour l'élaboration d'une séquence pédagogique. Quelles sont les postures épistémologiques face à la recherche des unes et de l'autre? Posture est définie ici comme la manière dont les enseignantes s'approprient le travail documentaire et se positionnent en tant qu'agent éthique. Cette posture épistémologique indique de quelle manière les protagonistes souhaitent créer de la connaissance. Quelles implications pour les différentes actrices? Quelles circulations de postures entre les actrices?

8 Je présenterai, dans un premier temps, les fondements et le dispositif de ce projet de recherche. Puis, je montrerai les conceptualisations opérées dans le cadre de la collaboration entre les praticiennes et la chercheure. Enfin, j'analyserai les différentes logiques des enseignantes et de la chercheure et je montrerai comment ces relations ont conduit à nous éclairer sur les spécifités de la méthodologie mise en œuvre. On pourra ainsi mettre en évidence l'intérêt de cette collaboration pour la formation des enseignants et l'avancée de la recherche.

\section{Méthodologie, contexte et corpus}

\section{Positionnement méthodologique}

9 Afin de mettre en lumière la réalité du travail documentaire des enseignants (recherche de ressources, sélection, didactisation...), une approche collaborative de recherche m'a semblé pertinente pour décrire des pratiques professionnelles 
(Desgagné, 1997 ; 1998 ; Desgagné et Bednarz, 2005 ; Bednarz, 2013). Cette démarche allie recherche et pratique en prenant en considération l'expérience acquise par les enseignants dans leurs tâches quotidiennes. Ces acteurs de la formation sont reconnus par le chercheur pour leurs compétences puisqu'ils connaissent l'environnement de leur pratique. Ils sont donc les plus à même d'élucider la question de recherche posée grâce aux savoirs produits (Bednarz, 2013). Mais cette démarche collaborative produit des effets pour les deux partenaires. En effet, si le chercheur peut répondre à la question de recherche posée, les enseignants, quant à eux, pourront développer une réflexion sur leurs pratiques, voire se former (Desgagné, 1998; Simonin et Thamin 2018). C'est ce que Dubet (1994) appelle le concept de "double vraisemblance» (cf. aussi Desgagné 1998 ; 2001). Cette réflexivité constitue le noyau de cette démarche collaborative lors des réunions avec les enseignants. Le dialogue généré entre les deux parties permettra de reconstruire l'expérience de la pratique (Desgagné, 2001) grâce à la confrontation des différentes expériences des protagonistes (enseignants et chercheur). Le chercheur accompagne l'explicitation des professeurs pendant la réflexion dans un échange dialogique ce qui permet ensuite, à partir d'une analyse des interactions entre chercheure et enseignantes, de catégoriser les éléments saillants. Cette «double vraisemblance» répond à une double pertinence sociale, une préoccupation de double rigueur méthodologique par la mise en place de l'activité réflexive et une préoccupation pour que les résultats soient féconds pour la recherche et la formation.

10 Dans le cadre de cette recherche, je me suis intéressée à la fois aux dimensions personnelle et sociale du rapport des enseignantes à leurs ressources pédagogiques. On note plusieurs tensions dans les pratiques documentaires en lien direct avec l'enseignement: prescriptions professionnelles, contraintes organisationnelles, situations d'enseignement et exigences normatives (Bento, 2017a). L'ensemble de ces éléments ont conduit à mettre en discussion les enseignantes sur le travail de transposition didactique effectué pour rendre les documents utilisés, souvent authentiques, accessibles aux élèves et comme leviers d'enseignement/apprentissage. On entend, par transposition didactique, les processus d'adaptation ou de transformation d'un contenu en un objet d'enseignement en vue de son apprentissage (Chevallard, 1985/1991; Bento, Defays, Meunier, 2014 : 10). Quelles didactisations sont mises en œuvre? Quelle organisation dans les séquences pédagogiques (Bento, 2017b) ? La question de l'éthique professionnelle a également été posée. Quels sont les moteurs de l'agir éthique des enseignantes quand on sait que l'expérience de la réalité de la classe résiste aux recommandations institutionnelles (Moreau, 2009: 78) ? Face à la déontologie professionnelle et à l'éthique déontologique, quelle position d'agent éthique les enseignantes adoptent-elles (Bento, 2017a)?

11 Au niveau de la recherche, ces questionnements ont été peu étudiés et il devenait intéressant de voir comment ils pouvaient être envisagés et développés lors d'une collaboration enseignantes/chercheure dans une démarche de double vraisemblance. Pour les enseignantes, cela leur permettait de réfléchir à leur démarche de documentation: quels documents, pour quoi faire, pour qui, quelle place dans la séquence, quelle transformation, quelle éthique; de mettre en discussion les enseignantes et de voir leur pratique respective ; de s'enrichir de la pratique des autres et d'échanger sur les différentes manières de faire. Du côté de la chercheure, cela lui 
permettait de mieux comprendre le travail documentaire des enseignantes et de situer ces analyses dans le paysage didactique.

\section{Le dispositif de collaboration}

12 Cinq professeures ont participé à cette recherche. Trois d'entre elles enseignaient dans des lycées généraux et technologiques du centre-ville d'Orléans et deux autres enseignaient dans un lycée professionnel de la banlieue proche d'Orléans (non classé en zone prioritaire).

La mise en place de cette recherche collaborative a pris pour cadre la démarche en trois étapes définie par Desgagné (1998) : la cosituation, la coopération et la coproduction.

La cosituation a pris la forme d'une négociation entre les enseignantes et la chercheure. Il s'est agi de se mettre d'accord sur les questions de recherche concernant le travail documentaire des enseignantes et sur le mode de collaboration lors des séances d'échange concernant les questions de recherche. C'est donc bien dans une démarche de double vraisemblance que l'activité réflexive a été envisagée. Il fallait créer des situations permettant de travailler les questionnements sur le travail documentaire en prenant à la fois en compte les préoccupations des cinq enseignantes et les objectifs de la chercheure.

15 L'étape de coopération a correspondu au moment de la collecte des données, objets d'enquête pour la chercheure et activités de réflexion pour les enseignantes. Les analyses croisaient diverses données recueillies à des moments, des lieux et auprès de différentes enseignantes. La recherche sur l'activité de documentation des enseignants était exploratoire et la démarche entreprise permettait d'augmenter la validité et la qualité des résultats obtenus (Silverman, 2009). Au préalable de la collaboration avec les enseignantes, il y a eu sept entretiens semi-directifs enregistrés, menés auprès des cinq enseignantes exerçant en lycée général et technologique et deux enseignantes exerçant en lycée professionnel. Le guide d'entretien était notamment composé d'une série de questions portant sur les données personnelles et professionnelles des enseignantes, leurs conceptions à propos des ressources pédagogiques utilisées en cours, leurs choix et leurs utilisations. La démarche de l'entretien semi-directif permettait la prise de parole spontanée des professeures interrogées et d'être attentive aux détails faisant sens pour elles. Ces entretiens ont donné lieu à une série de portraits d'enseignantes mettant ainsi en exergue les caractéristiques de chacune d'entre elles.

Le corpus était également constitué de journaux de bord de l'activité de trois enseignantes volontaires lors de la préparation des séquences pédagogiques. Elles notaient l'origine des ressources utilisées ou rejetées avec le cheminement qui avait présidé la recherche; l'intérêt des ressources retenues ou non; les modifications apportées aux ressources avec les techniques utilisées; l'organisation des ressources, mais aussi les activités organisées autour des ressources. Huit séquences pédagogiques ont pu ainsi être recueillies. C'est à partir de ces différents matériaux que le dialogue s'est établi lors de trois situations de collaboration qui prenaient la forme d'échanges collectifs sur les questions de l'activité documentaire des enseignants.

Ainsi, les entretiens individuels et les carnets de bord ont été présentés au groupe des cinq enseignantes volontaires, ayant participé à tout ou partie des phases du recueil des données (seulement trois enseignantes ont accepté de rédiger un carnet de bord), afin de déterminer ce qui se faisait réellement ou non. Les participantes réagissaient aux 
éléments présents dans les entretiens transcrits et les carnets de bord, elles confrontaient leurs conceptions et elles construisaient une culture commune. En effet, grâce à cette méthodologie, qui prenait en compte les savoirs d'expérience que les professeures développaient dans leur activité professionnelle journalière, une culture commune a émergé (Desgagné, 1998) axée sur les quelques règles qui régissent le métier de professeur d'anglais (Bento, 2017b).

Lors des rencontres, les enseignantes devaient donner du sens aux questionnements posés en prenant à la fois en compte et en commentant les transcriptions des entretiens, les journaux de bord et leur propre pratique. Il s'agissait principalement de repérer, à travers leurs activités quotidiennes, comment s'effectuait leur travail documentaire pour l'élaboration d'une séquence pédagogique. Il pouvait aussi s'agir pour les enseignantes de raconter une anecdote, un incident en lien avec la thématique. Des situations habituelles et inhabituelles d'activité étaient mises au jour afin d'en extraire les éléments les plus signifiants.

Enfin la dernière étape, la coproduction, a consisté en l'analyse des résultats. Chaque rencontre a été enregistrée et retranscrite. Le traitement des données a donné lieu à une analyse thématique du contenu des discours recueillis (Bardin, 2007). Il s'est agi de repérer les éléments significatifs du discours et des pratiques des enseignantes, puis de les catégoriser. Pour structurer l'analyse thématique de contenu, nous avons pris en compte plusieurs composantes susceptibles d'influencer le choix et la transformation des ressources par les enseignants.

\section{Recherche collaborative et conceptualisation des pratiques}

Cette démarche de collaboration guidait également la conceptualisation des pratiques qui étaient au cœur du projet et s'accordait avec le cadre des notions envisagées : le genre professionnel (Clot et Faïta, 2000) d'un côté et la déontologie professionnelle et l'éthique déontologique (Moreau, 2003 ; Prairat, 2009 et 2013) de l'autre.

\section{Le genre professionnel}

Le genre professionnel est défini comme

« un genre qui installe les conditions initiales de l'activité en cours, préalables de l'action [...]. Donné à recréer dans l'action, ces conventions d'action pour agir sont à la fois des contraintes et des ressources. Elles ont le caractère d'un prémédité social en mouvement qui ne relève pas de la prescription officielle mais qui la traduit, la « rafraîchit » et, si nécessaire, la contourne. Il existe des types relativement stables d'activités socialement organisées par un milieu professionnel au travers desquels le monde de l'activité personnelle s'accomplit, se précise, dans des formes sociales qui ne sont pas fortuites, ni d'un seul instant, qui ont une raison d'être et une certaine pérennité » (Clot et Faïta, $2000: 13$ ).

Le genre professionnel est donc perçu comme un élément du développement professionnel et Clot (1999) précise qu'il renvoie à des "schèmes sociaux d'utilisation » qui finissent par marquer la mémoire impersonnelle du collectif professionnel. Pour Cicurel (2013: 28), les enseignants alimentent le genre professionnel de leurs 
représentations idéales d'enseignement. Et pour Pastré, Mayen et Vergnaud (2006: 153), l'organisation de l'activité ne varie pas contrairement à l'activité :

«Cette organisation de l'activité est souple, puisque [...] la compétence ne consiste pas à répéter perpétuellement le même mode opératoire, mais à s'ajuster aux circonstances pour que l'action soit finement adaptée. Mais cet ajustement ne peut se concevoir que parce que l'organisation de l'activité comporte une bonne part d'invariance. La dimension invariante de l'organisation de l'activité représente la part généralisable de l'action. » dre aux questionnements posés: dégager la posture pédagogique des enseignantes quant à leur travail documentaire. Elles ont mis en évidence les conceptualisations qui leur permettent de réaliser des tâches documentaires compte tenu de leur formation, de leur expérience et des contraintes institutionnelles. Il a émergé aussi de la collaboration entre praticiennes et chercheure, l'importance des convictions professionnelles pour la construction du genre professionnel (Bento, 2017b), des convictions professionnelles partagées par la plupart des enseignantes. Mais cela ne voulait pas dire qu'il y avait uniformisation des pratiques. Des pratiques personnelles aussi ont émergé indiquant qu'au-delà du genre professionnel, il y a aussi un style (Clot, Faïta, 2000 : 15-16) qui renvoie à une « action singulière » (Cicurel, 2013 : 27).

\section{La déontologie professionnelle et l'éthique déontologique}

Pour Prairat (2009: 131), la déontologie professionnelle consiste à inventorier les obligations qui incombent à un professionnel dans l'exercice de sa tâche. Elle implique aussi des "restrictions normatives", issues des instructions officielles. L'éthique déontologique suppose qu'il y a des actions effectuées par les enseignants et d'autres non et ce, quelles qu'en soient les conséquences (Prairat, $2013: 38$ ). Ici, l'enseignant fait un choix pour être en accord avec lui-même quand il enseigne. Si la déontologie professionnelle s'applique aux règles, aux obligations et aux normes, l'éthique déontologique renvoie aux restrictions normatives (Prairat, 2013: 41) avec pour objectif le bien être de l'enseignant et l'intérêt de l'élève. Ces deux pans de la déontologie inscrivent l'enseignant comme un agent éthique: il prend une posture éthique face à son activité professionnelle. Cette activité professionnelle s'exerce en fonction d'un contexte institutionnel et social, mais aussi en fonction de l'histoire personnelle de l'enseignant (Bru, 2002).

La démarche méthodologique collaborative a montré les caractéristiques de l'éthique professionnelle des enseignantes centrée sur la déontologie professionnelle et l'éthique déontologique. Grâce au dialogue instauré, les raisons de l'agir éthique ont pu être 
dégagées. Ainsi, si les enseignantes tentent au maximum d'appliquer la méthodologie actuelle recommandée en didactique des langues dans les instructions officielles (approche actionnelle, recommandations du CECRL...), notamment l'usage de documents authentiques, elles peuvent renoncer à ces manières de faire si elles trouvent qu'elles vont à l'encontre des besoins ou du niveau des élèves ou encore de leurs goûts personnels (Bento, 2017a). Les interactions ont mis en évidence une exigence éthique de responsabilité personnelle. Les enseignants se positionnent en considérant qu'il y a des actes qu'elles peuvent faire ou ne pas faire. Ainsi,

« d'un côté les enseignants ne peuvent se passer des règles qui régissent le métier et constituent une culture commune, mais d'un autre côté, ces règles ne constituent pas à elles seules l'ensemble des conventions de la profession. La réalité des situations, l'intérêt des apprenants, le goût des enseignants sont autant d'éléments qui infléchissent l'activité des enseignants en la rendant singulière » (Bento, 2017a : 69)

\section{Enseignantes et chercheure : deux logiques complémentaires}

Comme nous l'avons vu plus haut, la recherche collaborative suppose une démarche de coconstruction pour répondre à un questionnement de la pratique (Desgagné : 1997). Les postures de chacune des partenaires sont donc symétriques et complémentaires. Chaque partie a une tâche dévolue différente. Les enseignantes prennent une posture de praticiennes réflexives. Elles questionnent leurs pratiques quant au travail documentaire qu'elles mettent en œuvre afin d'améliorer leur agir professionnel. La chercheure, quant à elle, va avoir une démarche d'investigation quant à ce même travail documentaire des enseignantes. Elle veille, par une démarche interactive, à construire avec les praticiennes la discursivité autour des savoirs mis au jour (Morrissette, 2013). Elle a été la médiatrice dans la démarche de coconstruction qui s'est mise en place et elle a interprété les discours (Savoie-Zajc et Descamps-Bednarz, 2007). De plus, la chercheure a veillé à ce que les actrices impliquées, elle comprise, ne portent pas de jugement normatif sur les pratiques décrites. Il s'est avant tout agi de comprendre les expériences de chacune pour un meilleur développement professionnel (Morrissette, 2013). La chercheure s'est présentée comme une facilitatrice dans la construction des connaissances. Cette collaboration laisse donc des traces dans les pratiques. L'extrait suivant, tiré du dernier échange, illustre une certaine évolution des pratiques.

\section{[...]}

Chercheure : Qu'est-ce qui fait que maintenant tu vas plus choisir ce texte plutôt qu'un autre? Parce qu'il te plait plus, parce que c'est plus adapté aux apprenants?

C : Au final parce que c'est plus adapté aux textes officiels.

Chercheure : Ça t'a rapprochée des instructions?

C: Euh, oui on pourrait dire ça, dans le côté global, on pourrait dire ça. En tous cas, je ne sais pas si ça m'en a rapproché, je pense que je faisais pareil sauf que je n'analysais pas pourquoi. Disons que je m'assure que je suis dans le cadre peut-être, davantage.

$[\ldots]$

S: Moi, je consulte davantage les manuels scolaires en fait. Ce que j'avais arrêté de faire il y a déjà un petit moment et puis on en a reçu quelques-uns. Je me dis «tiens ça fait un petit moment quand même que j'ai pas mis mon 
nez dedans » et finalement j'ai trouvé des choses intéressantes que j'avais laissé de côté alors que non il y a des choses intéressantes dans les manuels, donc oui je me suis mise récemment, je sais pas si toi (s'adresse à E.) tu les consultes autant

$\mathrm{E}$ : Euh oui, je les consulte. Voir s'il y a des idées de thèmes qui m'intéressent Chercheure : Mais tu le faisais déjà?

E : Oui, je le faisais déjà. Moi, je pense que c'est plus dans ma façon d'aborder le document avec les élèves. Je travaille plus la stratégie d'accès au sens. J'ai repris certains documents et ça m'a amenée à me poser des questions aussi sur pourquoi choisir tel document. $\mathrm{Y}$ a des documents que j'ai changé cette année en me disant que non par rapport à ce que moi je veux travailler. Si je veux travailler vraiment des stratégies d'accès au sens, vaut mieux prendre tel document que tel document donc peut-être pas sur beaucoup de chapitre mais sur... Ponctuellement, ça m'a amenée à changer soit le document, soit la façon d'aborder le document [...] (Transcription des échanges avec les 5 enseignantes participant à la recherche - 27 février 2017).

Cela étant, les professeures ne sont pas intervenues dans le travail formel de la recherche : définition du cadre théorique, méthodologie de recueil de données, analyse des données, production scientifique qui incombe aux chercheurs. Mais, la démarche entreprise a permis de mettre en œuvre un double volet, dans une perspective de double vraisemblance: l'examen du travail documentaire des enseignantes et le développement professionnel de ces mêmes enseignantes (Desgagné, 1997) en fonction de l'intérêt de chacune des parties.

Cependant, il serait faux de dire que la chercheure n'a pas influencé le travail de coconstruction élaboré. Par le fait qu'elle a dégagé des éléments saillants des différents corpus recueillis avant les rencontres, elle a orienté les discussions sur des questions qui lui semblaient pertinentes. Ainsi, ce sont les questions de genre professionnel et de déontologie qui ont été particulièrement développées. Desgagné (1997 : 373) signale que nous sommes face à une difficulté inhérente de la démarche collaborative. Cependant, c'est bien les dires des enseignantes qui ont orienté les choix de la chercheure. Ces choix reflètent les préoccupations saillantes des praticiennes lors des entretiens exploratoires. Il s'agissait donc de se soucier du contexte et des situations d'action des enseignantes. Ensuite, ensemble, elles sont allées chercher des pistes de compréhension de leur agir professionnel. Cette description des postures et de la circulation des postures qui s'est mise en place entre les actrices engagées montre la complexité de la démarche collaborative. La coopération doit être envisagée selon ses deux faces, chaque partenaire ayant besoin des expériences de l'autre. Marlot, ToullecThéry et Daguzon (2017:21) parlent d' "acculturation réciproque » où l'activité de chacun permet le développement de chaque acteur. Cependant ce développement ne se fait pas sans douleur, notamment pour les enseignantes. Elles sont d'accord pour dire que l'expérience est fructueuse, comme le montre cet extrait de mail écrit par une enseignante :

$\mathrm{E}$ : Ce projet a été vraiment formateur pour moi dans le sens où il m'a permis de mieux réfléchir sur ma pratique, mais également d'échanger avec des collègues d'ailleurs sur nos façons de faire et de voir les choses, et cela est toujours enrichissant. (Extrait du mail envoyé par E. le 18 avril 2018). suivant, tiré de la dernière séance, illustre bien le malaise engendré. 
C : Moi, depuis qu'on fait ce travail ensemble, je réfléchis au fait que je suis en train de rechercher une ressource et donc je pense à ce qu'on fait. C'est à dire le fait d'avoir réfléchi à ça. J'analyse ma recherche. C'est pas bien du tout. Ça prend encore plus de temps.

A : Dès qu'on commence à transformer une ressource on a l'impression d'avoir un gendarme au-dessus du nez qui nous dit «non » t'as pas le droit de faire ça. Là là non. C'est de l'authentique, t'es pas censée toucher ça. Et oui, c'est exactement ça, c'est le fait que quand on cherche une ressource, on pense bah au travail qu'on fait ensemble, aux échanges en fait.

Chercheure : Oui, ça vous facilite pas la tâche en fait?

$C$ : Non, ça nous facilite pas, pour moi en tout cas, j'ai pas l'impression que ça me facilite, simplement je réfléchis à ce que je fais et à ce que je faisais pas auparavant parce que j'allais chercher une ressource point alors que maintenant je me dis pourquoi est-ce que tu vas prendre plutôt cette cinquième apparition dans Google et non pas la deuxième. Qu'est ce qui fait que tu as tiré vers ça et non pas vers ça ? Voilà.

Chercheure : Et qu'est-ce que tu as comme réponse?

$\mathrm{C}:$ Je suis peut-être plus pointilleuse dans les mots, dans la petite synthèse qu'il y a. Je vais peut-être un peu plus loin qu'auparavant, tu vois. Mais je sais pas pourquoi.

Chercheure: Le travail qu'on fait ensemble il est pas, y a pas du tout de jugement. Par exemple, toi A., j'ai l'impression que tu culpabilises si tu n'as pas de document authentique

A : Ah non pas du tout.

Chercheure : alors qu'il n'y a aucun jugement en fait.

A : Non, c'est pas ça, c'est la réflexion qu'on apporte au travail qu'on fait et qu'on ne faisait pas auparavant et ça ça change. Ça ne nous demande pas plus de temps, c'est juste que ben dans notre tête y a un petit cheminement qui se fait sur effectivement comment je fais, sur quel moteur de recherche je vais, pourquoi je vais choisir effectivement cet article que cet article. Donc c'est juste une réflexion obligatoire en fait parce que voilà on relie quand même les éléments qui se passent dans notre vie et ça étant intégré maintenant à notre travail bah forcément on y pense. Mais c'est pas du tout péjoratif, loin de là, c'est juste que c'est rigolo.

C : Oui parce qu'auparavant c'était comme si c'était plus spontané en fait que maintenant. Je choisis mes vidéos pour les BTS, je vais réellement réfléchir. J'en ai trois devant moi et pourquoi je vais prendre celle-ci et pas ces deux-là et voilà je vais chercher davantage à identifier pourquoi mon choix va vers celle-ci que vers celle-là alors qu'avant j'allais vers la numéro $2, j$ 'allais vers la numéro 2 , je cherchais pas à savoir pourquoi j'avais éliminé la une et la trois, tu vois? C'est dans l'analyse de ce que je fais. (Transcription des échanges avec les 5 enseignantes participant à la recherche - 27 février 2017).

31 Ce long extrait montre le cheminement qui s'opère chez les praticiennes qui collaborent. Elles pensaient améliorer leurs pratiques, mais sans avoir évalué le poids que cela engendrerait en termes de questionnements. Si la recherche collaborative apporte réellement aux protagonistes, il n'en reste pas moins que cet apport n'est pas sans impact sur la vie professionnelle des enseignantes. Leurs tâches quotidiennes ne sont pas facilitées, elles se posent encore plus de questions et soupèsent chaque décision prise. 


\section{Conclusion}

Cet article présente une expérience de mise en œuvre de recherche collaborative entre cinq enseignantes d'anglais dans le secondaire et une chercheure autour de la question $\mathrm{du}$ travail documentaire et les aspects méthodologiques et théoriques liés à la recherche qui en découlent.

Outre le fait que cette recherche a permis de mieux appréhender le genre professionnel des enseignantes quant à leur travail documentaire au quotidien et de dégager les caractéristiques de leur éthique professionnelle et les raisons de l'agir éthique, elle a également permis de déterminer les postures épistémologiques des différentes actrices. On a vu aussi que ce travail collaboratif n'est pas si évident et qu'il peut produire une forme de malaise au travail dans le questionnement perpétuel qu'il enclenche chez les praticiennes.

Dans une démarche ultime, il ne faut pas oublier que l'approche collaborative, qui met au centre d'un côté les enseignants et les savoirs/savoir faire qu'ils construisent/ développent et de l'autre côté le chercheur qui produit des connaissances, a pour finalité l'apprentissage des élèves et que c'est en ce sens qu'oeuvrent les deux parties de la recherche.

\section{BIBLIOGRAPHIE}

Anadon, M. (2007). La recherche participative - Multiples regards. Québec : Presses Universitaires de Québec.

Bardin, L. (2007). L'analyse de contenu. Paris : Presses Universitaires de France.

Bednarz, N. (2013). Recherche collaborative et pratique enseignante : Regarder ensemble autrement. Paris : l'Harmattan.

Beckers, J. et Simons G. (2010). « Analyse rétrospective de programmes de recherches collaboratives en langues modernes à l'Université de Liège ». Recherches en éducation, hors-série 1 (Juin), 31-46.

Bento M., Defays J.-M. et Meunier D. (2014). « La transposition didactique du FLE et du FLS » Recherches et applications, $\mathrm{n}^{\circ}$ 55, 9-15.

Bento, M. (2017a). « Choix et transformation des ressources didactiques pour la classe d'anglais langue étrangère : une déontologie tempérée. » Etudes en didactique des langues, Focus sur l'enseignant.e, $\mathrm{n}^{\circ} 29,55-70$.

Bento, M. (2017b). « Le choix, la didactisation et l'organisation des ressources en anglais langue étrangère par les enseignants du secondaire en France : vers un genre professionnel. » Revue Méthodal, $\mathrm{n}^{\circ} 1,42-52$.

Bru, M. (2002). « Pratiques enseignantes : des recherches à conforter et à développer. » Revue française de pédagogie, $\mathrm{n}^{\circ} 138,63-73$. 
Castellotti, V. (2013). « L'articulation recherche-intervention en DDL : comment (ne pas) en sortir? ». Dans J.-C. Beacco (dir.), Éthique et politique en didactique des langues. Autour de la notion de responsabilité. Paris : Didier, 74-98.

Chevallard, Y. (1985/1991). La transposition didactique. Grenoble : La Pensée Sauvage.

Cicurel, F. (2013). « L'agir professoral entre genre professionnel, cultures éducatives et expression du « soi » ». Synergies Pays Scandinaves, $n^{\circ} 8,19-33$.

Clot, Y. (1999). La fonction psychologique du travail. Paris : PUF.

Clot, Y. et Faïta, D. (2000).« Genres et styles en analyse du travail. Concepts et méthodes ». Travailler, $\mathrm{n}^{\circ}$ 4, 7-42. Repéré à http://psychanalyse.cnam.fr/medias/fichier/ texteclot4_1306851012723.pdf.

Demaizière, F. et Narcy-Combes, J-P. (2007). « Du positionnement épistémologique aux données de terrain ». Les cahiers de l'Acedle, n 4. 1-20. Repéré à http://acedle.org/spip.php?article602.

Desgagné, S. (1997). « Le concept de recherche collaborative : l'idée d'un rapprochement entre chercheurs universitaires et praticiens enseignants. » Revue des sciences de l'éducation, $\mathrm{n}^{\circ} 23(2)$, 371-393.

Desgagné, S. (1998). « La position du chercheur en recherche collaborative : illustration d'une démarche de médiation entre culture universitaire et culture scolaire ». Recherches qualitatives, $\mathrm{n}^{\circ} 18,77-105$.

Desgagné, S. (2001).« La recherche collaborative : une nouvelle dynamique de recherche en éducation. » Dans M. Anadon (dir.), Nouvelles dynamiques de recherche en éducation. Québec : Les Presses de l'Université Laval, 51-76.

Desgagné, S. et Bednarz, N. (2005). « Médiation entre recherche et pratique en éducation : faire de la recherche « avec " plutôt que « sur » les praticiens. » Revue des sciences de l'éducation, $n^{\circ} 31(2), 245-258$.

Dubet, F. (1994). Sociologie de l'expérience. Paris : Éditions du Seuil.

Macaire, D. (2007a). « Didactique des langues et recherche-action. » Recherches en Didactique des Langues et Cultures : Les Cahiers de l'Acedle, $\mathrm{n}^{\circ}$ 4, p. 93-119.

Macaire, D. (dir.) (2007b). Notions en Questions : Méthodologies de recherche en didactique des langues. Recherches en Didactique des langues et des cultures : Les Cahiers de l'Acedle, $\mathrm{n}^{\circ} 4$. Repéré à http:// acedle.org/old/IMG/pdf/Macaire-D_cah4.pdf.

Marlot, C., Toullec-Théry, M. et Daguzon, M. (2017). « Processus de co-construction et rôle de l'objet biface en recherche collaborative » Phronosis, vol. 6, 21-34.

Moreau, D. (2009). « L'expérience éthique des enseignants débutants » Recherche et formation, $n^{\circ} 60,75-86$.

Morrissette, J. (2013). « Recherche-action et recherche collaborative : quel rapport aux savoirs et à la production de savoirs? » Nouvelles pratiques sociales, $n^{\circ} 25$ (2), 35-49.

Narcy-Combes, J.-P. (2005). Didactique des langues et TIC : vers une recherche-action responsable. Paris : Ophrys.

Pastré, P., Mayen, P., Vergnaud, G. (2006). « Didactiques professionnelles » Revue française de pédagogie, n 154, 145-198. Repéré à https://rfp.revues.org/157.

Silverman, D. (2009). Doing qualitative research. ( $3^{\text {rd }}$ ed.). London : Thousand Oaks \& New Delhi : Sage. 
Savoie-Zajc, L. et Descamps-Bednarz, N. (2007). « Action Research and Collaborative Research : Their Specific Contributions to Professional Development. » Educational Action Research, vol. 15, $n^{\circ} 4,577-596$.

Simonin, M.-C. et Thamin, N. (2018). « Recherche collaborative à l'école maternelle et socialisation plurilingue. » Diversité, n 192, La recherche en éducation. Vers de nouvelles interfaces, 131-136.

\section{NOTES}

1. Le projet ReVEA porte sur les utilisations de ressources par des enseignants du secondaire en France. Centrée sur les critères de sélection des ressources, il apparaît que cette recherche permet aussi d'approcher les questions posées par les processus de professionnalisation des enseignants.

\section{RÉSUMÉS}

À partir des résultats d'une étude portant sur l'usage des ressources pédagogiques par des enseignants du secondaire en France, cet article présente une expérience de mise en œuvre de recherche collaborative entre une chercheure et cinq enseignantes d'anglais dans le secondaire sur leur travail documentaire. Les résultats mettent en évidence les logiques des enseignantes et de la chercheure orientées pour les unes sur le développement professionnel et pour l'autre sur la production de connaissances.

Based on the results of a study on the use of learning resources by secondary school teachers in France, this article presents an experience of implementing collaborative research between a researcher and five English secondary school teachers on their documentation activity. The results highlight the logic of teachers and researchers who are oriented respectively towards professional development and knowledge production.

\section{INDEX}

Mots-clés : recherche collaborative, travail documentaire, éthique professionnelle, genre professionnel, didactique de l'anglais

Keywords : collaborative research, documentation activity, professional ethics, professional style, English didactics

\section{AUTEUR}

\section{MARGARET BENTO}

Université de Paris - Faculté Sciences et Humanités

Laboratoire Éducation, Discours, Apprentissages 
Margaret Bento est professeure en sciences du langage spécialisée en didactique des langues étrangères et maternelles. Elle s'intéresse tout particulièrement aux méthodologies

d'enseignement des langues.

margaret.bento[at]parisdescartes.fr 